\title{
Primera cita de Trifolium vesiculosum Savi (Fabaceae) en la provincia de Sevilla (Andalucía, España)
}

\section{Javier López-Tirado, Vicente Rodríguez-Estévez, Cipriano Díaz-Gaona \& Santos Sanz-Fernández}

Departamento de Producción Animal, Facultad de Veterinaria, Universidad de Córdoba, Campus de Rabanales, 14071 Córdoba (España)

\section{Correspondencia}

J. López-Tirado

e-mail: javier.lopez.tirado@uco.es

Recibido: 21 septiembre 2020

Aceptado: 16 octubre 2020

Publicado on-line: 12 noviembre 2020

Editado por: Marta Recio Criado

\begin{abstract}
Resumen
Se cita por primera vez la leguminosa Trifolium vesiculosum Savi para la provincia de Sevilla.
\end{abstract}

Palabras clave: Corología, Fabaceae, Trifolium vesiculosum, Sevilla.

\begin{abstract}
First record of Trifolium vesiculosum Savi (Fabaceae) in Seville province (Andalusia, Spain)

The legume Trifolium vesiculosum Savi is recorded for the first time in Seville province.
\end{abstract}

Key words: Chorology, Fabaceae, Trifolium vesiculosum, Seville.
Trifolium vesiculosum Savi, FI. Pis. 2: 165 (1798) SEVILLA. Cazalla de la Sierra: Finca El Coronado [30STH5307], 655 m, 17-IX-2020, J. López Tirado s.n. (COA 60503).

Trifolium vesiculosum Savi es un trébol cuya área de distribución se extiende por el sur de Europa e islas del Mediterráneo hasta Turquía y Crimea (Muñoz et al., 2000). En Portugal aparece disperso en gran parte de sus regiones (https://flora-on.pt/; último acceso Septiembre 2020) y en España es también errático y escaso, estando presente en las Islas Baleares (Mallorca y Menorca), en las provincias de Barcelona y Gerona (Muñoz et al., 2000; FragaArguimbau et al., 2018), en la de Cádiz (Sánchez García, 2004), en la de Córdoba (López-Tirado, 2016) y en las de Huelva, Badajoz, Tarragona y Toledo (de acuerdo con las citas recogidas en ANTHOS (Sistema de información sobre las plantas de España, http://www.anthos.es/; último acceso Septiembre 2020). También se cultiva en países sudamericanos como Chile (Ovalle et al., 2010). Esta especie tiene gran parecido con T. mutabile (DC.) Kuntze, por lo que puede confundirse, si bien se diferencian por la morfología del cáliz y la presencia o no de nervios transversales en el mismo (Muñoz \& Devesa, 1988).

Recientemente, se ha encontrado esta especie en una dehesa mixta de encina (Quercus rotundifolia Lam.) y alcornoque ( $Q$. suber $L$.) en la provincia de Sevilla, para la que constituye novedad corológica.

\section{Agradecimientos}

Esta especie ha sido localizada en el marco de los trabajos de campo del proyecto LIFE LiveAdapt (LIFE17 CCA/ES/000035) cofinanciado por la Unión Europea a través del programa LIFE.

\section{Bibliografía}

Fraga-Arguimbau, P., Mascaró-Sintes, C., PallicerAllès, X. \& Carreras-Martí, D. (2018). Notes i contribucions al coneixement de la flora de Menorca (XIII). Notes florístiques. Bolletí de la Societat d'Història Natural de les Balears, 61, 183198.

López-Tirado, J. (2016). Sobre la presencia de Trifolium vesiculosum Savi (Fabaceae) en la provincia de Córdoba (Andalucía, España). Botanica Complutensis, 40, 71-73.

Muñoz, A. \& Devesa, J.A. (1988). Trifolium sect. Mystyllus (C. Presl) Godron en España. In Homenaje a Pedro Monserrat. Instituto de Estudio Altoaragoneses, Instituto Pirenaico de Ecología (C.S.I.C.).

Muñoz, A., Devesa, J.A. \& Talavera, S. (2000). Trifolium L. In S. Castroviejo et al. (Coord.), Flora Iberica 7(II) (pp. 646-719). Madrid: C.S.I.C.

Ovalle, C.M., del Pozo, A., Fernández, F., Chavarría, J. \& Arredondo, S. (2010). Arrow leaf clover 
(Trifolium vesiculosum Savi): A new species of annual legumes for high rainfall areas of the Mediterranean climate zone of Chile. Chilean Journal of Agricultural Research, 70(1), 170-177.
Sánchez García, Í. (2004). Nota sobre algunas especies nuevas para la flora gaditana. Revista de la Sociedad Gaditana de Historia Natural, 4, 239241. 